\title{
Simulation of Smith-Purcell terahertz radiation using a particle-in-cell code
}

\author{
J. T. Donohue \\ Centre d'Etudes Nucléaires de Bordeaux-Gradignan, BP 120, 33175 Gradignan, France \\ J. Gardelle
}

CEA CESTA, BP 2, F-33114 Le Barp, France

(Received 14 February 2006; published 12 June 2006)

\begin{abstract}
A simulation of the generation of Smith-Purcell (SP) radiation at terahertz frequencies has been performed using the two-dimensional particle-in-cell code MAGIC. The simulation supposes that a thin (but infinitely wide) monoenergetic electron beam passes over a diffraction grating. We simulate two configurations, one similar to the Dartmouth SP free-electron laser, with a low-energy continuous beam (we use an axial magnetic field to constrain the electrons to essentially one-dimensional motion). The other is similar to the recent MIT experiment that uses a prebunched $15 \mathrm{MeV}$ beam.
\end{abstract}

DOI: 10.1103/PhysRevSTAB.9.060701

PACS numbers: $41.60 . \mathrm{Cr}, 41.75 . \mathrm{Ht}$

\section{INTRODUCTION}

In a recent publication [1], we reported on a simulation of coherent Smith-Purcell (SP) radiation using a twodimensional particle-in-cell (PIC) code, MAGIC. The grating period and the beam energy were chosen such that the radiation produced was in the microwave region, with frequency of order $10 \mathrm{GHz}$. We remind the reader of the SP relation, $\lambda=L(1 / \beta-\cos \phi) / n$, where $\lambda$ is the wavelength, $L$ the grating period, $\beta$ the relative velocity (in a plane parallel to the grating and perpendicular to the direction of the grooves), $\phi$ the angle of emission (with respect to the beam direction), and the integer $n$ denotes the order. Our aim was to verify the analytical model proposed by Andrews and Brau [2] and subsequently extended by Andrews, Boulware, Brau, and Jarvis [3] to explain coherent Smith-Purcell radiation. Since our previous experience with the code had been in the microwave domain, we preferred to work there. Our results do indeed support the viewpoint of Brau and co-workers, that the mechanism for coherent radiation is the bunching of the initially continuous beam by an evanescent wave that is significant only in the vicinity of the grating. The frequency of this wave is always less than the minimum allowed SP frequency. The process is unstable in the sense that the wave bunches the beam, the beam drives the wave and growth occurs, both in time and along the grating. Our simulation found that this is what happens. In particular, the frequency and axial wave number (in the first Brillouin zone) of the simulated grating wave were very close to what the model predicts. However, it also revealed two major effects that had not been anticipated. The first was copious emission of radiation at the evanescent frequency. Since our simulated grating has a finite length, when the evanescent wave reaches the end, part of its energy is emitted as free radiation of the same frequency, and part of it is reflected back in the opposite direction. This happens at both ends of the grating. The second unforeseen effect was the presence of spatial oscillations in $B_{z}(x)$ just above the grating with two different wave numbers, $k_{+}$and $k_{-}$, while the current $I(x)$ contained only $k_{+}$. The new wave number satisfied the condition, $k_{+}+k_{-}=K=2 \pi / L$. As a result of discussions at the conference, in particular, with Brau [4], and benefiting from suggestions of the referee, we now claim to understand what is going on. We shall explain this in Sec. III with the aid of the dispersion relation for the evanescent wave.

To the best of our knowledge, no observation of this copious radiation, whose frequency is below that for allowed SP emission, has been reported by the various groups studying SP radiation experimentally. However, the two-dimensional nature of our simulation requires a beam of infinite width in the direction of the grooves. In practice, we imagine that beam at least several wavelengths wide would be necessary, but the existing experiments typically use narrow round beams. Thus, confirmation of the results of our simulation and the validity of the Andrews and Brau model will need dedicated experiments with wide beams.

The main interest in SP free-electron lasers (FELs) is the possibility they offer of being compact, powerful, and tunable sources of $\mathrm{THz}$ radiation. An example is the ongoing Dartmouth College experiment [5], initiated by Walsh many years ago, and often reported on in this series of conferences. This experiment typically uses optical gratings of period $0.17 \mathrm{~mm}$, and beam energies in the range $30-50 \mathrm{keV}$. To reach $\mathrm{THz}$ frequencies only the lowest SP orders are needed. Quite recently a new direction was opened by a group at MIT [6] using the beam from a linac operating at $17.14 \mathrm{GHz}$, that delivers pulses of duration 1 ps. The beam energy was $15 \mathrm{MeV}$, and the grating period was $1 \mathrm{~cm}$. Here the $\mathrm{THz}$ domain requires high orders near $90^{\circ}$. An important issue in both of these experiments is coherence. According to Ref. [3], there are two distinct concepts involved, intrabunch and interbunch coherence. The former obtains whenever the physical size of an electron bunch is small compared to the wavelength of the 
radiation observed. Then the fields created by each electron in the bunch add up, and the total power radiated will be proportional to the square of the number of electrons per bunch. This occurs for all wavelengths such that the ratio of bunch size to wavelength is $\ll 1$. Interbunch coherence requires, in addition to the bunch-size criterion, that the contributions of successive bunches add coherently at the observation point. This occurs whenever the frequency of the bunches (inverse of the time interval between two successive bunches) is equal to the frequency of the SP radiation. This is never true for the fundamental, but since bunching is nonlinear, higher harmonics may also appear in the current. When this happens coherent SP radiation may occur at angles $\phi$ and order $n$ such that $m f_{\text {ev }}=$ $c n / L(1 / \beta-\cos \phi)$. In this expression $f_{\mathrm{ev}}$ is the frequency of the evanescent wave, $m$ is an integer, and $c$ denotes the speed of light.

In the Dartmouth experiments, with an initially continuous beam, the intersection of the beam line and the grating dispersion relation determines the operating point, i.e., the frequency of the evanescent wave. In contrast, in the MIT experiment, the beam is already bunched when it reaches the grating, and the Andrews-Brau operating point is not relevant. Since the beam pulses are periodic in time, interbunch coherent SP radiation occurs whenever the angle and order are such that the frequency is an integer multiple of the repetition frequency, namely, $17.14 \mathrm{GHz}$. A thorough discussion of the radiation emitted by prebunched beams has been given recently by Gover [7].

The paper is organized as follows: Sec. II contains a description of the particle-in-cell code and the details of the simulation. In Sec. III we show our results for the Dartmouth experiment, while in Sec. IV we display those for the MIT experiment. Brief conclusions are given in Sec. V, and a calculation of radiation associated with the turning on of a narrow pulsed sheet beam is presented in the Appendix.

\section{DETAILS OF THE SIMULATIONS}

The simulations are performed using the commercially available code MAGIC, a 2D/3D electromagnetic PIC code, i.e., a finite-difference, time-domain code for simulating plasma physics processes. Beginning from a specified initial state, the code simulates a physical process as it evolves in time. The full set of Maxwell's time-dependent equations is solved to obtain electromagnetic fields. Similarly, the complete Lorentz force equation is solved to obtain relativistic particle trajectories, and the continuity equation is solved to provide current and charge densities for Maxwell's equations. Our version of the code is two dimensional; it assumes that all fields and currents are independent of the $z$-coordinate. However, the motion of electrons is calculated in three dimensions.

In Fig. 1, we display the geometry we have chosen for our 2D Cartesian simulation, where the electron beam

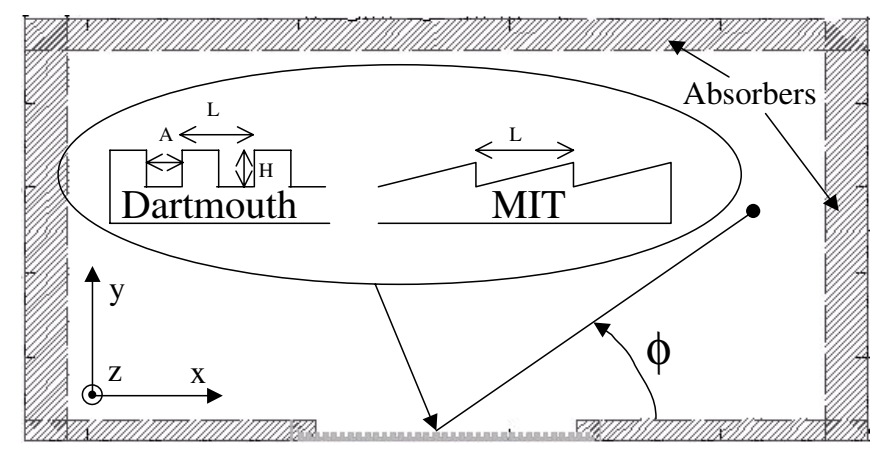

FIG. 1. Simulation geometry. Details of the gratings are shown in the inset.

TABLE I. Parameters of the simulations.

\begin{tabular}{lll}
\hline \hline Parameters & Dartmouth & MIT \\
\hline Beam energy & $50 \mathrm{keV}$ & $15 \mathrm{MeV}$ \\
Current (peak for MIT) & $1000 \mathrm{~A} / \mathrm{m}$ & $25 \mathrm{kA} / \mathrm{m}$ \\
Beam thickness & $\delta=20 \mu \mathrm{m}$ & $1 \mathrm{~mm}$ \\
Beam-grating distance & $e=20 \mu \mathrm{m}$ & $0.7 \mathrm{~mm}$ \\
Grating period & $L=173 \mu \mathrm{m}$ & $1 \mathrm{~cm}$ \\
Maximum wave number & $K=363 \mathrm{~cm}^{-1}$ & $2 \pi \mathrm{cm}^{-1}$ \\
Grating groove depth & $H=100 \mu \mathrm{m}$ & \\
Grating groove width & $A=62 \mu \mathrm{m}$ & \\
Number of periods & $N=35$ & 10 \\
External magnetic field & $B_{x}=2 \mathrm{~T}$ & 0 \\
Mesh size & $(10 \mu \mathrm{m})^{2}$ & $(100 \mu \mathrm{m})^{2}$ \\
\hline \hline
\end{tabular}

propagates along the $x$-direction. The setup includes a perfectly conducting grating in the center at the bottom, a small cathode, which emits beam, and a vacuum box in which radiation propagates. The boundary consists of absorber, shown crosshatched, which prevents most of the radiation that reaches the walls from reflecting back into the box. We have verified that this feature of MAGIC greatly suppresses reflection, at least for nearly normal incidence.

The choice of observable quantities in the code is quite rich: electric and magnetic field components as a function of time at a point in space, or as functions of space at fixed time. The electron phase space, current, and the Poynting vector may also be displayed. Our uniform density electron beam is thin in the $y$-direction and infinite in the $z$-direction. For the Dartmouth simulation the beam leaves the cathode steadily, while for the MIT simulation a square-wave pulse of current of duration 1 ps is emitted at a frequency of $17.14 \mathrm{GHz}$. The MIT grating is of the échelette type, while that of Dartmouth is laminar. We summarize the simulation parameters in Table I.

\section{RESULTS OF THE DARTMOUTH SIMULATION}

In Fig. 2 is shown the dispersion relation for the grating in the Dartmouth simulation, along with the light line and 


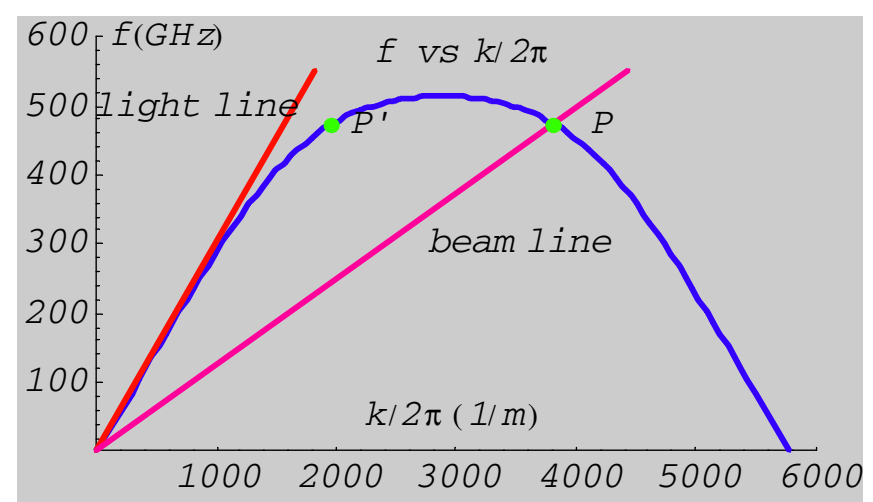

FIG. 2. (Color) Dispersion relation for the Dartmouth grating according to Andrews and Brau. The operating point $P$ is shown, as well as a second point $P^{\prime}$ which has the same frequency but wave number $k^{\prime}=K-k$. The solutions $P$ and $P^{\prime}$ go into each other upon reflection.

the beam line for $50 \mathrm{keV}$ electrons. The operating point $P$ is indicated, with $f=473 \mathrm{GH} z, k_{+} / 2 \pi=3824 \mathrm{~m}^{-1}$, i.e., the intersection of the dispersion relation with the beam line, $f=\nu k / 2 \pi$. At $P$, the group velocity $2 \pi d f / d k$ is negative, which means that electromagnetic energy flows upstream, as in the backward wave oscillator (BWO). According to Floquet theory, the $x$-dependence of the fields just above the grating is of the form $e^{i k_{+} x} u(x)$, where the function $u(x)$ is periodic under $x \rightarrow x+L$. This solution has an arbitrary scale factor, but the ratios of all components are fixed by the solution of Maxwell's equations. If a field variable is measured as a function of $x$ for fixed $y$ and $t$, the corresponding Fourier transform will display peaks at wave numbers $k_{+}+n K$, where $n$ is any integer. However, since the field variable is real, the Fourier transform will also have identical peaks at $-\left(k_{+}+n K\right)$. But these may be written as $K-k_{+}-(n+1) K$, a series of equidistant peaks which includes the positive wave number $k_{-}=K-$ $k_{+}$. It turns out that $k_{-}$is the wave number corresponding to the point $P^{\prime}$ shown in Fig. 2, which corresponds to a solution of the dispersion relation with the same frequency. Having observed a large Fourier component of the magnetic field near the grating with this wave number in Ref. [1], we erroneously suggested that this second solution was being anomalously generated, probably by reflections at the ends of the grating, and we expressed surprise at its importance. The discussion presented here shows our interpretation was wrong. Even if there is no contribution from the second solution, the large Fourier component at $k_{-}$is expected. Although experts certainly were well aware of it, we only recently learned that the reflection of a Floquet solution with wave number $k_{+}$is just the solution with $k_{-}$. Thus a certain amount of the evanescent wave with $k_{+}$is reflected at the grating ends, and does appear as the $k_{-}$solution. A future goal for us is to quantify the relative amounts of both waves in our simulation, which involves looking at the dependence of the fields on both space and time.

In Fig. 3, a contour map of the magnetic field components $B_{z}$ and $B_{y}$ in the $x-y$ plane at time $t=0.9 \mathrm{~ns}$ is displayed. In fact, these are composite maps, each of 2$\mathrm{mm}$ width in $y$ and joined together afterwards, to maintain color contrast. Although in the ideal case there is only a $z$-component of the magnetic field, the small transverse motion of the electrons gives rise to very small $x$ and $y$ components as well. While the contour map for $B_{z}$ is complex, dominated mainly by copious emission of radiation at the evanescent frequency from both ends of the grating (of wavelength $0.635 \mathrm{~mm}$, visible on the left), the map of $B_{y}$ indicates radiation of half that wavelength emerging at a well-defined angle of approximately $55^{\circ}$. Guided by this, one can discern the same thing in the $B_{z}$ map, although it is masked by the presence of the radiation at the evanescent frequency. This is the second harmonic of the evanescent frequency, which is emitted coherently at $54^{\circ}$. We point out that the current used in our simulation, $1000 \mathrm{~A} / \mathrm{m}$ in a thin sheet beam, is quite large compared to current densities realized in practice. We chose such a large value to speed up the long computations.

In Fig. 4 we show $B_{z}(t)$ at angle $50^{\circ}$ and distance $4.5 \mathrm{~mm}$ from the center of the grating, along with finite Fourier transform (FFT) of the signal. This distance is much greater than the attenuation length of the evanescent wave, and we are clearly in the radiation zone. (But we are not far enough away for the SP correlation between angle and frequency to hold rigorously.) The time signal

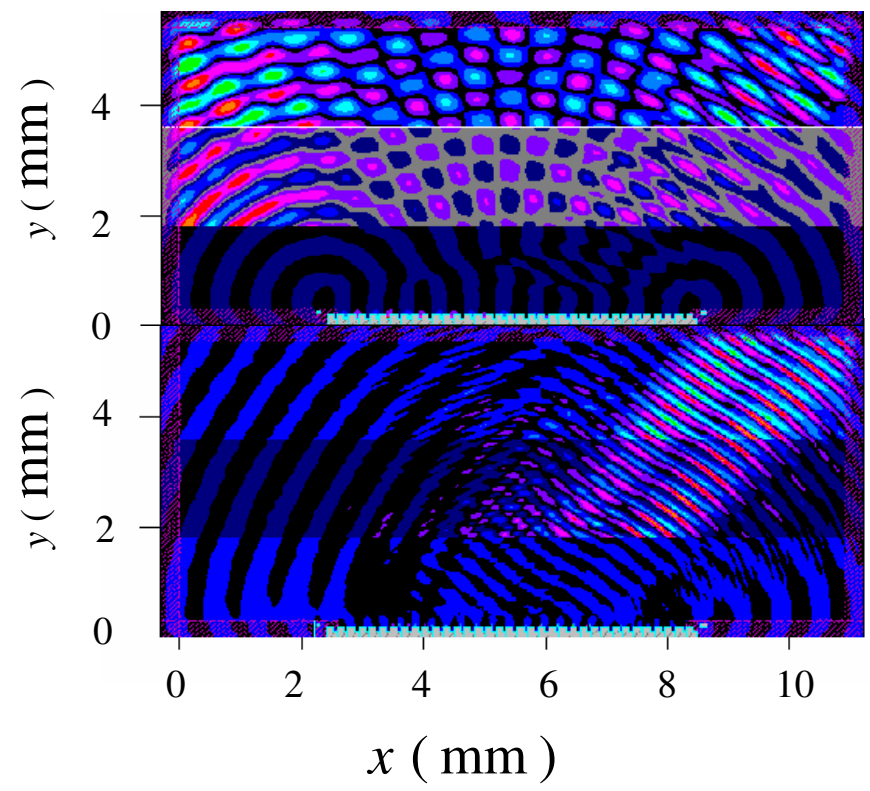

FIG. 3. (Color) $x-y$ contour maps of $B_{z}$ (upper) and $B_{y}$ (lower) (Dartmouth). The latter shows clear evidence of coherent SP radiation at twice the evanescent frequency, which may then be recognized in the former, amidst the radiation at the evanescent frequency emitted at the grating ends. 

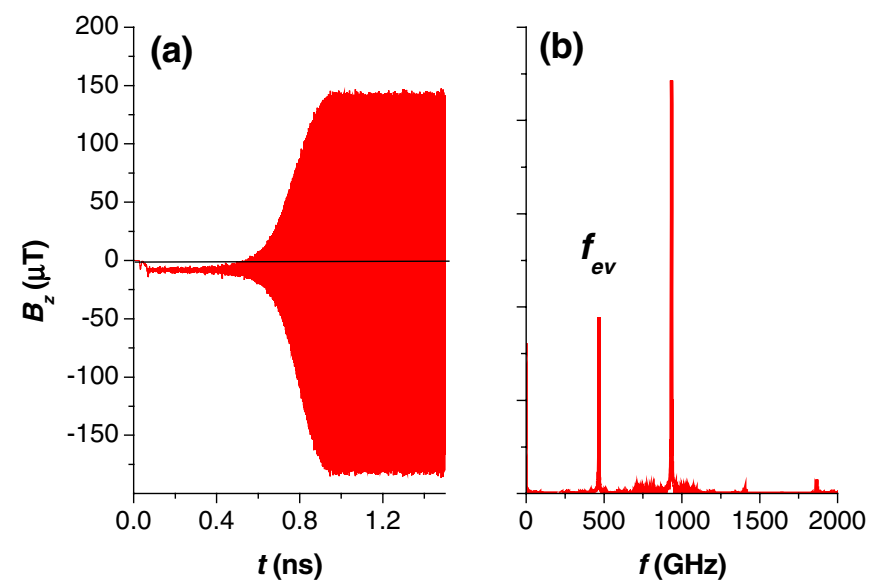

FIG. 4. (Color) Magnetic field (a) $B_{z}(t)$ and (b) the corresponding FFT at a detector placed at $50^{\circ}$ and $4.5 \mathrm{~mm}$ from the grating center (Dartmouth).

shows rapid growth after $0.5 \mathrm{~ns}$. The frequency of the evanescent wave is indicated, and one sees that at this angle the second harmonic is the dominant frequency. Tiny amounts of third and fourth harmonics are also visible. Note that the fourth harmonic in second order should be coherent at the same angle as the second harmonic in first order.

In Fig. 5 we show a snapshot of the beam just above the grating, along with its distribution in $T-x$ phase space, where $T$ denotes the kinetic energy. Strong bunching is apparent in both. By counting oscillations we estimate approximately $0.25 \mathrm{~mm}$ for the wavelength, quite consistent with the value expected from the dispersion relation. We also note that the mean energy loss is $1-2 \mathrm{keV}$ or $2 \%$ to $4 \%$ of the beam energy.

To illustrate the nature of the evanescent wave, we show in Fig. 6(a) the quantity $B_{z}$ vs $x$ at fixed time $(0.9 \mathrm{~ns})$ and at a $y$ position just above the grating. The corresponding FFT

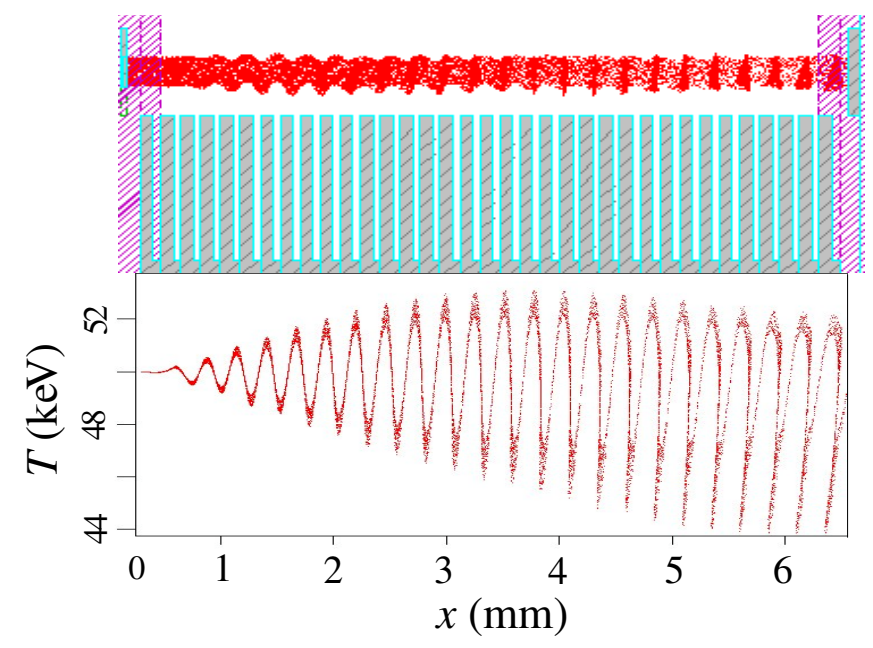

FIG. 5. (Color) Section of beam above grating (upper), and $T-x$ phase-space distribution (lower) (Dartmouth).

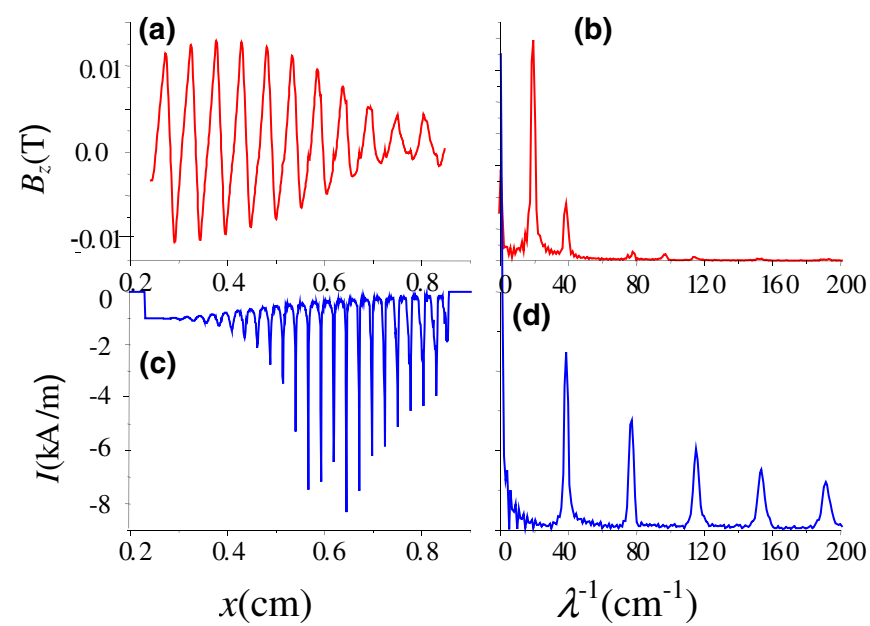

FIG. 6. (Color) Space dependence of magnetic field and current: (a) $B_{z}(x)$ near grating, (b) corresponding FFT, (c) $I(x)$, (d) corresponding FFT (Dartmouth).

is shown in Fig. 6(b). For comparison, the quantity $I(x)(I$ has dimension of $A /$ meter) is displayed in Fig. 6(c), with its FFT in Fig. 6(d). It is clear that the behavior of these two quantities is quite different. The spatial modulation of the current increases with increasing $x$, while that of the magnetic field decreases. The visible period of the oscillations in $B_{z}$ is about $0.5 \mathrm{~mm}$, quite different from that of the current, but entirely consistent with $k_{-}$in the Andrews and Brau dispersion relation. As we stated above, the corresponding wave number is $K-k_{+}$, which is the negative of an allowed component of the Floquet wave. The FFTs confirm this picture, and illustrate the difference between the two. For the magnetic field, Floquet theory implies that the Fourier spectrum (with the convention of the code MAGIC) should show peaks at $k_{+} / 2 \pi+n / L$, where $n$ denotes an integer $\left(1 / L=57.80 \mathrm{~cm}^{-1}\right)$. There are also peaks at the negatives of these values, which amounts to peaks at $k_{-} / 2 \pi+n / L$. Such behavior is evident in Fig. 6(b), where the two principal peaks, at 19 and 38.8, are accompanied by their translations of $1 / L$ which appear at 77 and 96, respectively. In contrast, the beam is bunched by the component of the longitudinal electric field that behaves like $e^{i\left(k_{+} x-\omega t\right)}(\omega=2 \pi f)$. Since bunching is inherently nonlinear, the higher spatial and temporal harmonics appear when it becomes strong, as in Fig. 6(c). This explains why only lines at integer multiples of $k_{+} / 2 \pi$ are present in the FFT of $I(x)$, Fig. 6(d). We point out that it is an accident that some of these peaks coincide with those in 6(b). This stems from the fact that for $50 \mathrm{keV}$ beam energy, $k_{+} \cong \frac{2}{3} K, k_{-} \cong \frac{1}{3} K$, so the Floquet recurrences should occur at (approximately) $\left\{\frac{1}{3}, \frac{2}{3}, \frac{4}{3}, \frac{5}{3}, \frac{7}{3}, \frac{8}{3}\right.$, $\left.\frac{10}{3}, \frac{11}{3}, \ldots\right\} L^{-1}$. The harmonic peaks are at $\left\{\frac{2}{3}, \frac{4}{3}, \frac{6}{3}\right.$, $\left.\frac{8}{3}, \frac{10}{3}, \frac{12}{3}, \ldots\right\} L^{-1}$, which has four in common with the Floquet series. In the generic case, the only coincidence would be between the first peak of the current and the second of the magnetic field. 


\section{RESULTS OF THE MIT SIMULATION}

In the MIT experiment, the beam consists of intense very short bunches repeated at $17.14 \mathrm{GHz}$. Consequently, the radiation field is periodic in time, and only integer multiples of $f_{0}=17.14 \mathrm{GH} z$ are observed. Because the highenergy beam does not undergo appreciable deflection in passing over the grating, we include no magnetic field in our simulation. Noting that the wavelength corresponding to the fundamental frequency is $1.749 \mathrm{~cm}$, we find that coherent SP radiation should be emitted at a large number of frequencies and angles. If we are interested in the $n$th harmonic of $f_{0}$, the standard formula assures us that it is emitted at angles $\phi_{m n}$, where $\cos \phi_{m n}=\frac{1}{\beta}-\frac{m c}{n f_{0} L} \cong 1-$ $1.749 \frac{m}{n}$. We show in Fig. 7 the intersections of the six lowest orders $(m=1, \ldots, 6)$ and the first 17 harmonics, for angles from 0 to $90^{\circ}$. At each intersection of an order curve with a horizontal harmonic line, coherent SP radiation may occur. In fact, at some angles several harmonics may be coherent. A subset of these angles are indicated by the vertical lines. Given the complexity of this figure, it is clear that extremely accurate measurements of angle and frequency are needed to unravel coherent SP radiation in this experiment.

In Fig. 8 we show two contour plots of $B_{z}$ in the $x-y$ plane at fixed time, $1.7 \mathrm{~ns}$. In the upper figure there is no grating, but rather a $10-\mathrm{cm}$ long flat ground plane, while the lower has an échelette grating. The upper map is not SP radiation. Indeed, in the limit of zero beam thickness and neglecting minor effects of the cathode and beamstop, we can write the quantity $B_{z}(x, y, t)$ in closed form using standard electrodynamics (see the Appendix). The lower map obviously bears an overall resemblance, but has a much richer spatial structure, since true SP radiation oc-

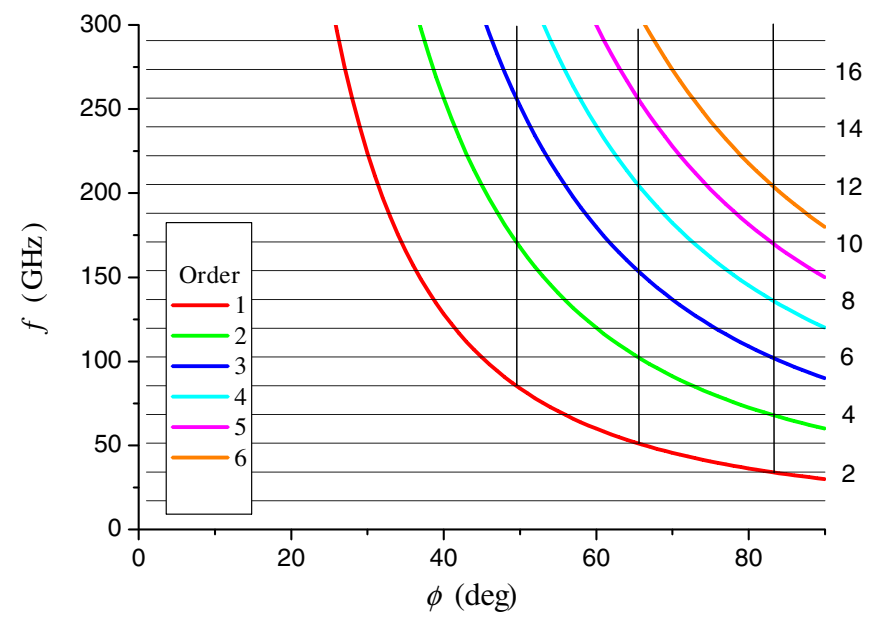

FIG. 7. (Color) Frequency $f(\mathrm{GHz})$ vs emission angle $\phi\left({ }^{\circ}\right)$ for the lowest six orders of SP radiation in the MIT experiment. Coherent SP radiation is expected at angles where an order curve intersects a harmonic (indicated at right) of the bunch frequency, $17.14 \mathrm{GHz}$.

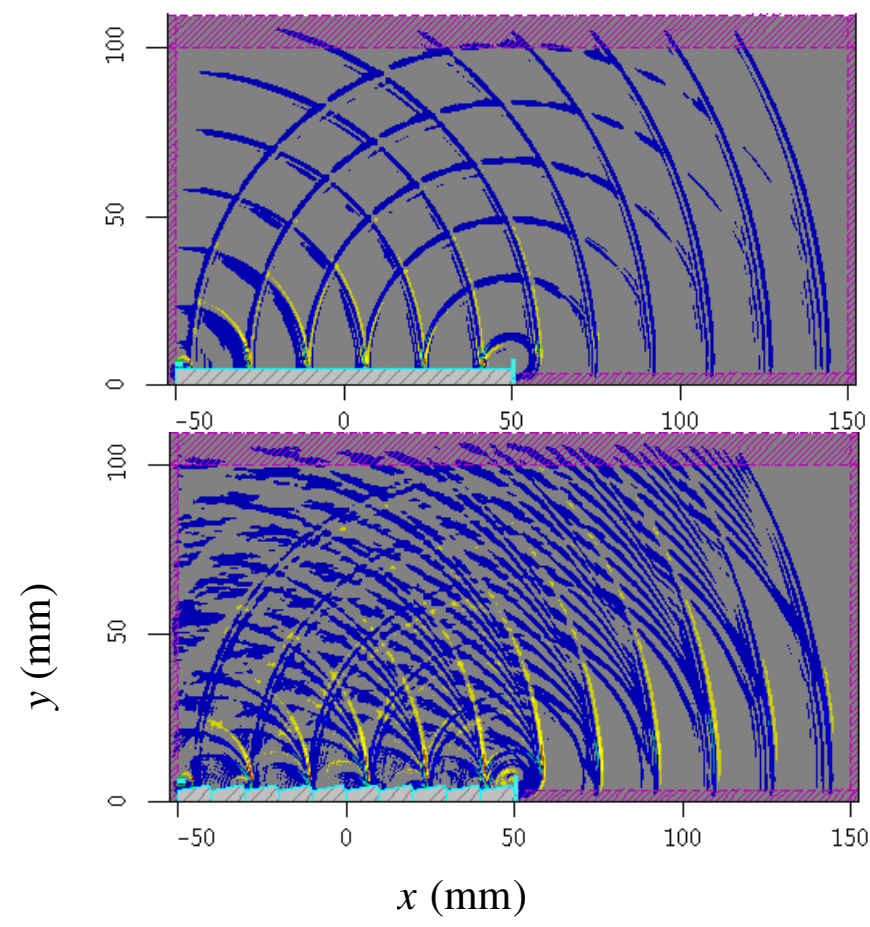

FIG. 8. (Color) $B_{z}$ contour maps with a flat ground plane (upper) and with a grating (lower) (MIT).

curs. In order to quantify the difference, we show in Fig. 9 $B_{z}(t)$ at $\phi=48^{\circ}$ for both cases, along with their FFTs. The distance from the grating center is $10 \mathrm{~cm}$. At $48^{\circ}$, the lowest SP frequency is $90 \mathrm{GHz}$; the second order is 180 . The sixth and twelfth harmonics, at 102 and $204 \mathrm{GHz}$, would be SP coherent at $45^{\circ}$. This may account for their strong presence in the FFT. Some caution should be exercised in interpreting these results, since our simulation box may be too small (compared to the grating length) to allow the field to assume its radiation zone limit. A simu-

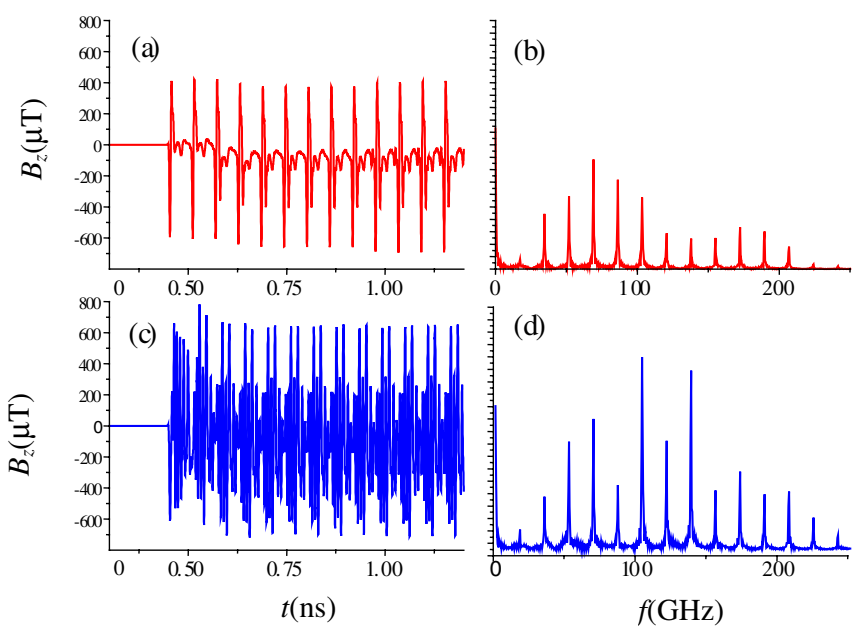

FIG. 9. (Color) Magnetic field at $48^{\circ}$ : (a) $B_{z}(t)$ and (b) its FFT with a flat ground plane. The same, but with the grating, are shown in (c) and (d) (MIT). 
lation with a larger box but sufficiently small mesh size is probably required to address this question.

\section{CONCLUSIONS}

We have presented results concerning simulations of coherent Smith-Purcell radiation, using a 2D PIC code. Both of the experiments we attempt to simulate use narrow round beams, and it is not prudent to claim that all of the phenomena we predict should be seen experimentally. Given this caveat, we find that our Dartmouth setup simulation lends support to the model of Brau and co-workers, while the simulation of the short-pulse MIT experiment suggests that an interpretation only in terms of coherent SP radiation may oversimplify the true situation. The observation of radiation even without a grating is accounted for by the calculation provided in the Appendix.

Quite recently two articles addressing the question of coherent SP radiation at $\mathrm{THz}$ frequencies have appeared. Kumar and Kim have performed a detailed 2D analysis in which the SP free-electron laser is treated as a BWO [8], while a PIC code simulation of the Dartmouth experiment has been presented by Li et al. [9]. In the former, emphasis is placed on deliberately extracting power at the sub-SP frequency of the evanescent wave, rather than using harmonics of that frequency at the SP angles. In the latter, the simulation indicates that the passage of a single short pulse can excite the evanescent wave, which then escapes from the grating ends after the pulse has moved on. In addition, by using a periodic train of short pulses, the authors avoid exciting the evanescent wave, and are able to clearly observe coherent SP radiation at harmonics of their imposed bunching frequency. These results, in addition to our own, offer support to the Andrews and Brau model of coherent $\mathrm{SP}$ radiation.

\section{APPENDIX}

Radiation emitted when an intense relativistic electron beam is emitted or absorbed has been discussed in the 1970s, notably by Longmire [10]. Although a charge in uniform motion does not radiate, the appearance and disappearance of a beam leads to short pulses of radiation. In the two-dimensional context of our simulation of the MIT experiment, with an infinitely long line charge, standard electrodynamics provides an exact answer for the magnetic field. This can be extended to include a sheet beam pulse of finite duration, in the limit of zero thickness. While the exact answer is somewhat complicated, the result for the radiation part of the field is rather simple. Although this may be well known to specialists in the field, we present it here to interpret the results of our simulation without grating.

First, we find the magnetic field in the $z$-direction for an infinitely long line charge moving at uniform speed along the $x$-axis through a finite distance $L$. The current density may be written using the Dirac $\delta$-function,

$$
J_{x}(x, y, z, t)=\frac{q \nu}{l} \delta(y-h) \delta(x-\nu t), \quad-\frac{L}{2} \leq x \leq \frac{L}{2},
$$

where the charge per unit length is $q / l$, and the velocity is denoted $\nu$. The origin of the time scale is chosen such that the line charge is at $x=0$ when $t=0$. Following the steps outlined in Jackson [11] (Sec. 14.1), one obtains the vector potential $A_{x}$ (in Gaussian units, with line charge in statC/cm, and $B$ in Gauss) and from it the $z$-component of the magnetic field, which we call $B_{\text {line }}$ :

$$
\begin{aligned}
B_{\text {line }}(x, y, t)= & {\left[\frac{2 q \gamma \beta(y-h)}{l\left\{[\gamma(x-\nu t)]^{2}+(y-h)^{2}\right\}}\right] } \\
& \times\left[\theta\left(t-t_{1}\right)\left(\frac{\gamma\left[t-t_{1}+(1-\beta \cos \psi) R / c\right]}{\sqrt{\left(t-t_{1}\right)\left(t-t_{1}+2 R / c\right)}}\right)\right. \\
& -(L \rightarrow-L)],
\end{aligned}
$$

where

$$
\begin{gathered}
\beta=\nu / c, \quad \gamma=1 / \sqrt{1-\beta^{2}}, \\
R=\sqrt{(x+L / 2)^{2}+(y-h)^{2}}, \quad \cos \psi=(x+L / 2) / R, \\
t_{1}=-L / 2 \nu+R / c .
\end{gathered}
$$

The term in brackets is the field of a line charge moving along $x$ from $-\infty$ to $+\infty$. The standard Heaviside function $\theta$ assures that the field is zero for $t<t_{1}$, where $t_{1}$ is the instant when a light signal emitted when the beam was turned on reaches the observation point $(x, y)$. The corresponding distance (in a plane perpendicular to the line charge) is denoted $R$. The contribution from the disappearance of the beam is represented by the $(L \rightarrow-L)$, which means replacing $L$ by $-L$ in the definitions of $R, \psi$, and $t_{1}$.

Next we consider a sheet beam, with a square-wave pulse form of duration $\tau$, where the current density is given by $(q / l$ now represents the charge/length of the pulse)

$$
\begin{aligned}
J_{x}(x, y, z, t) & =\frac{q}{l \tau} \delta(y-h)[\theta(x-v t)-\theta(x-v t-\nu \tau)], \\
-\frac{L}{2} & \leq x \leq \frac{L}{2} .
\end{aligned}
$$

The magnetic field is just the superposition of the field for the line charge

$$
\begin{aligned}
B_{\text {sheet }}(x, y, t) & =\frac{1}{\tau} \int_{0}^{\tau} d \tau^{\prime} B_{\text {line }}\left(x, y, t-\tau^{\prime}\right) \\
& =\frac{2 q}{l c \tau}\left[\theta\left(t-t_{1}\right) \arg (\Omega)-(L \rightarrow-L)\right]
\end{aligned}
$$

where the complex quantity we call $\Omega$ is defined by (henceforth we denote $t-t_{1}$ by $\hat{t}$ ): 
for $0 \leq \hat{t} \leq \tau$

$$
\begin{aligned}
\Omega= & i \gamma \beta \sin \psi+\frac{\gamma\left[R\left(1-i \gamma \beta^{2} \sin \psi \cos \psi\right)+\beta(R+c \hat{t})(i \gamma \sin \psi-\cos \psi)\right]}{\sqrt{c \hat{t}(2 R+c \hat{t})}} \\
& +\frac{\gamma^{2}\left[R(R+c \hat{t})(1-\beta \cos \psi)^{2}-c^{2} \hat{t}^{2} \beta \cos \psi\right]}{c \hat{t}(2 R+c \hat{t})}
\end{aligned}
$$

while for $\tau \leq \hat{t}$

$$
\Omega=\frac{1+\frac{c \hat{t}(\gamma \cos \psi+i \sin \psi)+(\cos \psi+i \gamma \sin \psi)\{\sqrt{c \hat{c}(2 R+c \hat{t})}-\sqrt{c(\hat{t}-\tau)[2 R+c(\hat{t}-\tau)]}}{\gamma \beta R-(\cos \psi+i \gamma \sin \psi) \sqrt{c \hat{t}(2 R+c \hat{t}}-(\gamma \cos \psi+i \sin \psi)(R+c \hat{t})}}{1-\frac{c \tau \gamma \beta}{c \hat{t} \gamma \beta+R[\gamma(\beta-\cos \psi)-i \sin \psi]}} .
$$

To include the effect of a ground plane, we add an image beam of opposite charge at $y=-h$. The final result for a sheet pulse passing over a ground plane is

$$
B_{\text {sheet, ground }}(x, y, t)=\frac{2 q}{l c \tau}[\{\theta(\hat{t}) \arg (\Omega)-(L \rightarrow-L)\}-\{h \rightarrow-h\}] .
$$

Although our complex-valued function $\Omega$ is complicated, it simplifies considerably in the limit of large $R$. We find

$$
\begin{array}{ll}
0 \leq \hat{t} \leq \tau, & \arg \Omega \approx \arctan \left(\frac{\sin \psi}{1 / \beta-\cos \psi} \sqrt{\frac{2 c \hat{t}}{R}}\right) \\
\tau \leq \hat{t}, & \arg \Omega \approx \arctan \left[( \frac { \operatorname { s i n } \psi } { 1 / \beta - \operatorname { c o s } \psi } \sqrt { \frac { 2 c \tau } { R } } ) \left(\sqrt{\frac{\hat{t}}{\tau}}-\sqrt{\left.\frac{\hat{t}}{\tau}-1\right)}\right.\right.
\end{array}
$$

This behavior is indeed simple at distances large compared to $L, h$, and $c \tau$. First the field increases as the square root of $\hat{t}$, then a cusp occurs at $\hat{t}=\tau$, followed by a decrease which is asymptotically proportional to $\sqrt{\tau / \hat{t}}$.

In the far-field zone, one may make the following approximations:

$$
\begin{aligned}
& R \rightarrow \rho=\sqrt{x^{2}+y^{2}}, \quad \psi \rightarrow \arctan (y / x), \\
& \hat{t} \rightarrow t-\frac{\rho}{c}+\frac{L}{2 c}\left(\frac{1}{\beta}-\cos \phi\right)-\frac{h}{c} \sin \phi .
\end{aligned}
$$

This means that the four contributions are identical, to within a time translation and sign differences. It should be noted that for highly relativistic electrons and small angles the four pulses arrive almost at the same time, making the signal very short and hence dominated by high frequencies. Finally, to take into account a train of periodically spaced bunches, as does our simulation, one writes, with $T$ denoting the bunch spacing in time,

$$
B_{\text {bunch train }}(x, y, t)=\sum_{n} B_{\text {sheet,ground }}(x, y, t+n T) .
$$

In this way the Fourier spectrum becomes discrete, and our formulas may be integrated numerically for frequencies $f=m / T$, where $m$ is an integer. In general, we find that the results of our simulation are in good agreement with the formulas given here, although the presence of the conducting cathode and beamstop causes some minor differences.

[1] J. T. Donohue and J. Gardelle, Phys. Rev. ST Accel. Beams 8, 060702 (2005).

[2] H.L. Andrews and C.A. Brau, Phys. Rev. ST Accel. Beams 7, 070701 (2004).

[3] H.L. Andrews, C.H. Boulware, C.A. Brau, and J.D. Jarvis, in Proceedings of the 2004 FEL Conference, Trieste, Italy, p. 278 (http://www.JACoW.org).

[4] C. A. Brau (private communication).

[5] A. Bakhtyari, J. E. Walsh, and J. H. Brownell, Phys. Rev. E 65, 066503 (2002).

[6] S. E. Korbly, A. S. Kesar, J. R. Sirigiri, and R. J. Temkin, Phys. Rev. Lett. 94, 054803 (2005).

[7] A. Gover, Phys. Rev. ST Accel. Beams 8, 030701 (2005).

[8] Vinit Kumar and Kwang-Je Kim, Phys. Rev. E 73, 026501 (2006).

[9] D. Li, Z. Yang, K. Imasaki, and Gun-sik Park, Phys. Rev. ST Accel. Beams 9, 040701 (2006).

[10] C. L. Longmire, Mission Research Corporation Report, MRC-R-315, 1977.

[11] J. D. Jackson, Classical Electrodynamics (John Wiley \& Sons, New York, 1975), 2nd ed. 